\title{
The sound of light: photoacoustics for biomedical applications
}

Hilde Jans and Xavier Rottenberg

imec, Kapeldreef 75, 3001 Leuven, Belgium

Looking inside the body

Medical imaging techniques provide a unique view inside the body and are invaluable for diagnosis and disease monitoring. From X-ray, through magnetic resonance imaging (MRI) to ultrasound, the field is vast and diverse. When imaging biological tissue, the choice of the modality depends on the contrast used for imaging and the tradeoff between resolution and depth. Light waves can generate highresolution images but do not travel far unperturbed. Deeper in tissue, the light gets scattered resulting in blurry images. High-energy X-rays form a special case as they penetrate deep into the tissue and yield high-resolution images, but their ionising radiation limits their use.

To circumvent these drawbacks, other options that do not rely on unperturbed light propagation have been explored as well. Acoustic or sound waves are well known to safely monitor foetuses in the womb using ultrasound imaging. These mechanical waves are less scattered than electromagnetic waves of comparable frequencies or wavelengths so they can

\section{DOI: 10.1255/sew.2022.ax}

(C) 2022 The Authors

Published under a Creative Commons BY-NC-ND licence

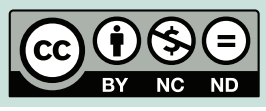

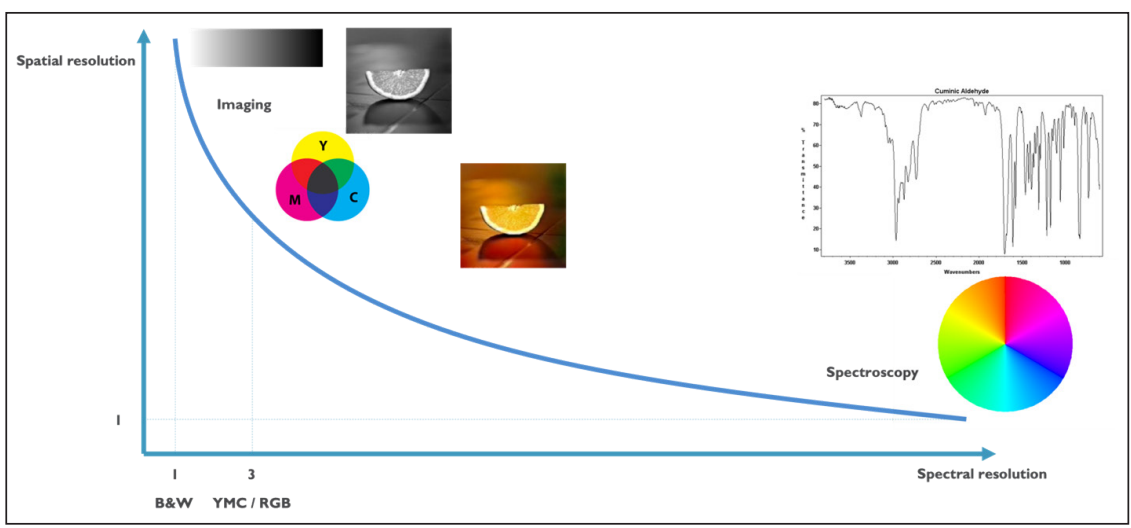

Figure 1. Imaging versus spectroscopy. While imaging explores a larger area in a few spectral components (blue, red, yellow), spectroscopy visualises the whole spectral signature of one component.

reach objects deeper in the tissue. However, ultrasound images typically suffer from a low resolution. MRI, based on radio waves that interact with hydrogen nuclei, shows similar features with a good depth exploration but limited resolution. MRI images have greater detail than ultrasound images but they are typically not real-time and are static. Moreover, MRI is a cumbersome technique that often requires contrast agents to enhance resolution.

In the soft spot between these established imaging methods emerges a novel technique with the resolution of light-based imaging and the good depth penetration of sound-based imaging, called photoacoustics (PA). It is capable of imaging finer blood vessels than other techniques without the need for contrast agents or X-ray exposure (photoacoustic imaging, PAI). PA can also be applied for spectroscopy that describes the spectral characteristics of an object when light interacts with it (photoacoustic spectroscopy, PAS), for example, to identify biomolecules and monitor their concentrations based on a unique spectral signature (Figure 1). Imec is currently working on the technology to unlock the full potential of PA for biomedical applications.

\section{The sound of light}

PA combines light and sound to create an image based on the photoacoustic effect first discovered by Alexander Graham Bell over a century ago. Bell noticed that certain materials emit sound when struck by pulses of light. Absorption of the light causes the molecules in those materials to heat up. Heat in 


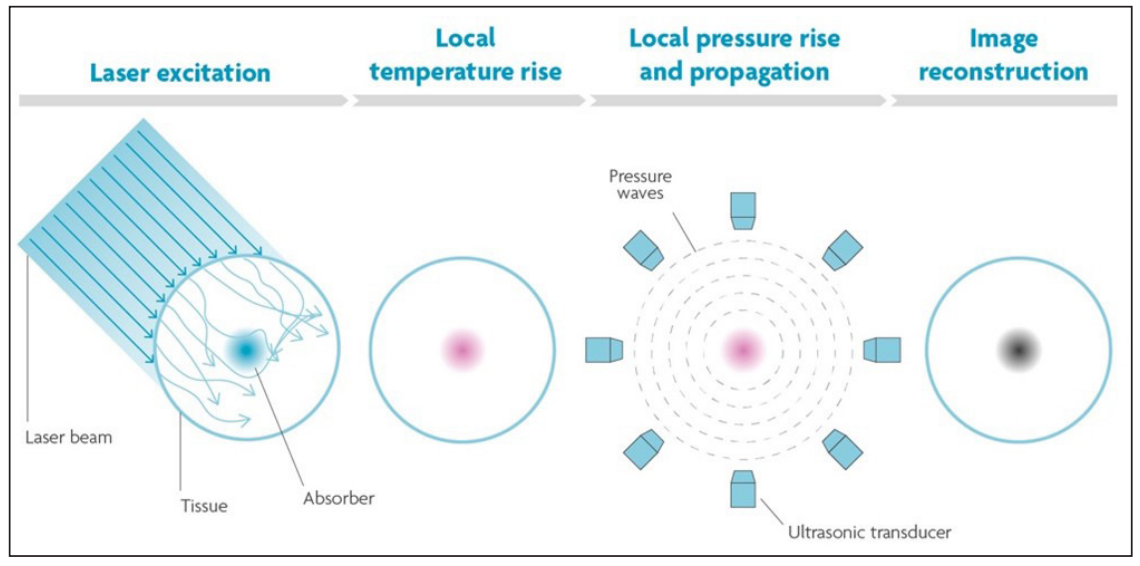

Figure 2. The photoacoustic principle. When pulses of light hit tissue, the molecules that absorb the light will expand and relax due to heat. These vibrations cause a pressure wave that can be detected and reconstructed to an image.

turn produces pressure changes when the molecules expand and relax and push against the surrounding tissue. This pressure or sound wave can be detected by a (array of) microphone(s) and reconstructed to a high-resolution image (Figure 2).

The advantage of PA is that it does not focus with light-which gets attenuated in the tissue-but with sound. Only the target molecule or structure that absorbs the light will selectively send pressure waves. That means that "optical contrast" images can be achieved at deeper locations and in turbid structures. There is also no need for fluorescent labels or tagging. By tuning the wavelength of the laser beam the contrast of target structures can be enhanced or by using different wavelengths different structures can be visualised in one image. An interesting application is the detection of oxygen saturation levels in blood haemoglobin, where oxygenated and deoxygenated haemoglobin absorbs at different wavelengths. These characteristics apply for spectroscopy as well, leading to a technique with zero background and a very low limit of detection. When light is shone onto a sample, it will only send out acoustic waves if the slightest quantity of a particle is present and absorbs the light.

\section{High-sensitivity ultrasound microphones}

Semiconductor technologies enable sensitive microphones and light sources with high spectral purity onto a chip, bringing PA sensing to the next level. On the detectorside, a large, dense array of highsensitivity ultrasound microphones with high-bandwidth readout is required. The signals in PA are very small because of tissue attenuation. The more sensitive and low noise the microphone is, the deeper in the tissue you can listen. Imec's current opto-mechanical ultrasound sensor is considered bestin-class for photoacoustic and ultrasonic imaging. ${ }^{1}$ It is based on an opto-mechanical waveguide, rather than a piezoelectric crystal, to convert sound to a measurable optical signal (Figure 3). The novel approach results in a detection limit two orders of magnitude better than state-of-the-art piezoelectric elements of identical size. This enables applications like through-skull functional brain imaging, where the pressure waves are very small because of the strong ultrasound attenuation of bone. Moreover, a fine-pitched matrix the integration of both ultra- of these tiny sensors can be easily integrated on-chip with photonic multiplexers, opening up the way to new applications such as miniaturised catheters.

\section{Light to create sound}

For imaging purposes, light sources typically have one or a few wavelengths. The target structure will absorb light at a preferred wavelength. A second or third wavelength can then be used complementarily to create a background for the target structure. Imaging light sources are high power to guarantee sufficient energy density to generate an image in a large volume of tissue $\left(\right.$ about $1 \mathrm{~cm}^{3}$ ). Finally, they need to be able to pulse light. A single thermal expansion of a molecule will not give rise to a pressure wave. For that to happen, the molecule also has to relax back. It is the alternating expansion and relaxation that arises when you pulsate the light, that creates a detectable sound wave.

For spectroscopy, the requirements are a bit different. In this case, you need a tuneable light source, or a light source with a broad wavelength range that you can modulate to generate the acoustic signal. However, the technique often requires the acquisition of many separate images at each wavelength of interest, which prolongs imaging time and creates errors when the sample moves between acquisitions. A dual comb laser would constitute an elegant solution for this issue and is, therefore, under research for PA applications.

An optical frequency comb simultaneously generates thousands of discrete optical frequency bands that are evenly spaced and very narrow, just like the teeth of a comb (Figure 4). In a dual comb source, two combs are combined, one with the frequencies slightly shifted compared to the other. Pairs of comb teeth, one from each comb, 


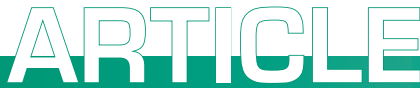
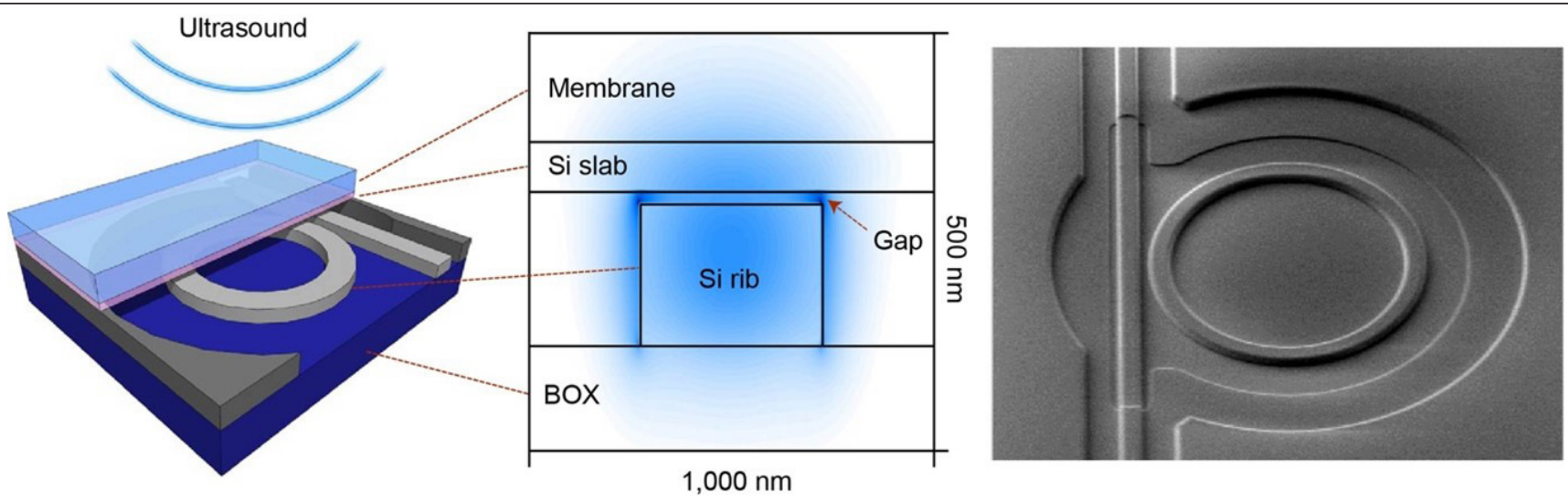

Figure 3. Cross-section and SEM image of imec's opto-mechanical ultrasound sensor.

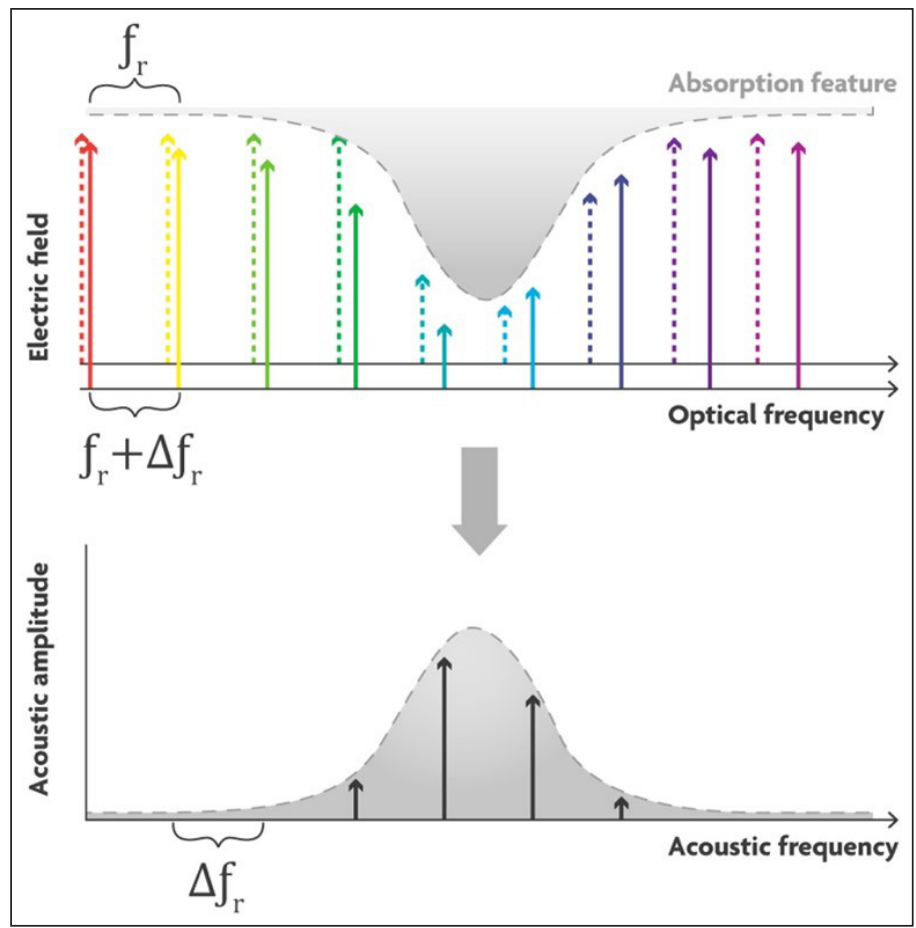

Figure 4. Principle of a dual-comb light source. Two frequency combs with slightly different optical frequency bands interact to generate beating. Microphones can detect the beat notes on a unique frequency when the light is absorbed.

interfere with each other resulting in "beating". The beat notes are detected by the microphone. The average optical frequency of each pair is modulated with a unique acoustic frequency, in other words, the optical absorption spectrum is copied into the acoustic domain. For example, for the "green" wavelength comb pair, the average green will be absorbed by the target molecule and produce a unique tone with a frequency equal to the difference between the two "green" optical frequencies. If the microphone picks up a signal at the green acoustic frequency, you can see a spectral peak at that frequency.

Imec together with the Photonics Research Group, an imec research group at Ghent University, recently created a mode-locked laser-the most popular light source to generate a dual comb-that can be integrated on chip (Figure 5). ${ }^{2}$ On-chip integration opens the possibility of miniaturised, stable and low-cost laser sources. Current demonstrations on a silicon platform have shown a limited performance regarding pulse energy, noise and stability due to relatively high waveguide losses and temperature sensitivity of the platform. Imec's integrated mode-locked laser is fabricated on silicon-nitride (SiN). $\mathrm{SiN}$ is one of the main photonic integration platforms that features very low waveguide loss and low temperature sensitivity compared to, for example, silicon. The result is a first step towards highpulse-energy, low-noise, on-chip mode-locked lasers that imec is researching as a candidate for dualcomb PA spectroscopy.

\section{The spectrum of light sources}

The dual-comb laser is the Rolls Royce of light sources but not all applications require such a fancy light source. $\mathrm{CO}_{2}$, for example, has such a large absorption peak at $4.3 \mu \mathrm{m}$ that it can be detected with a simple black body radiator that emits a broadband, continuous spectrum. $\mathrm{CO}_{2}$ sensing is rather an exception; a good spectrometer for 


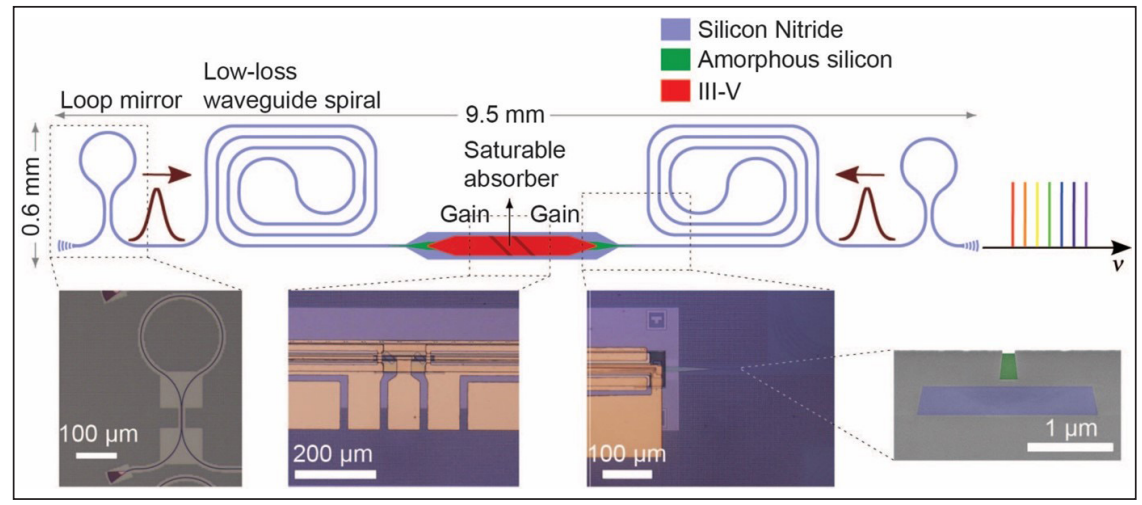

Figure 5. Schematic figure of the mode-locked laser on SiN designed by imec and the Photonics Research Group of Ghent University.

complex sensing of similar components still requires a good light source with narrow spectral bandwidth, such as arrays of quantum cascade lasers or the dual-comb lasers. Aside from these high-end sources, imec is also working on mid-end light sources based on light emitting diodes (LEDs). LEDs are interesting candidates for both imaging and spectroscopy because they are low-cost, robust and easy to use. The challenge with LEDs, on the other hand, is that they do not produce a spectrum right away which a dual comb laser does. By combining two to six LEDs you can already achieve a rough spectrum. Though resolving the absorption peak will be difficult, with correlation of background and other processing techniques it is possible. Current work in imec is focusing on an array of LEDs on-chip in the visible range.

\section{Applications}

PAI and PAS are emerging as new, non-invasive techniques for biomedical applications that fill the gaps between existing modalities (Figure 6). PAl is particularly well-suited to image blood vessels and oxygen saturation, since haemoglobin has a strong PA signature. Hence, the diagnosis of tumours, which often show neovascularisation, is a potential application domain for PAI. In particular, PAl is being investigated as an alternative for mammography. Mammography is the primary inspection method today for breast cancer. However, it can be painful, involves exposure to $X$-rays and still shows difficulties in detecting tumours in dense breast tissue. PA can reach depths $>5 \mathrm{~cm}$, does not use harmful radiation and can clearly show new networks of vessels around the tumour by tuning the light source to the absorption frequency of haemoglobin. Other imaging applications include functional brain imaging, detection of arteriosclerosis and retinal imaging.
PAS can be employed to detect biomarkers in blood such as cortisol, or for breath analysis. The Holy Grail, however, is non-invasive blood glucose sensing which is essential for diabetes patients. It is a challenging application because the glucose signal is often weak due to the differences in human skin and changes the skin undergoes depending on the environment. Once you have a robust glucose sensor, you would also be able to learn about glucose metabolism and how the concentration changes because the signal will be stronger when glucose concentration is higher. And most importantly, finger-pricking would be a thing of the past.

\section{References}

1. W.J. Westerveld, Md. MahmudUI-Hasan, R. Shnaiderman, V. Ntziachristos, X. Rottenberg, S. Severi and V. Rochus, "Sensitive, small, broadband and scalable optomechanical ultrasound sensor in silicon photonics", Nature Photonics 15, 341-345 (2021). https:// doi.org/10.1038/s41566-02100776-0

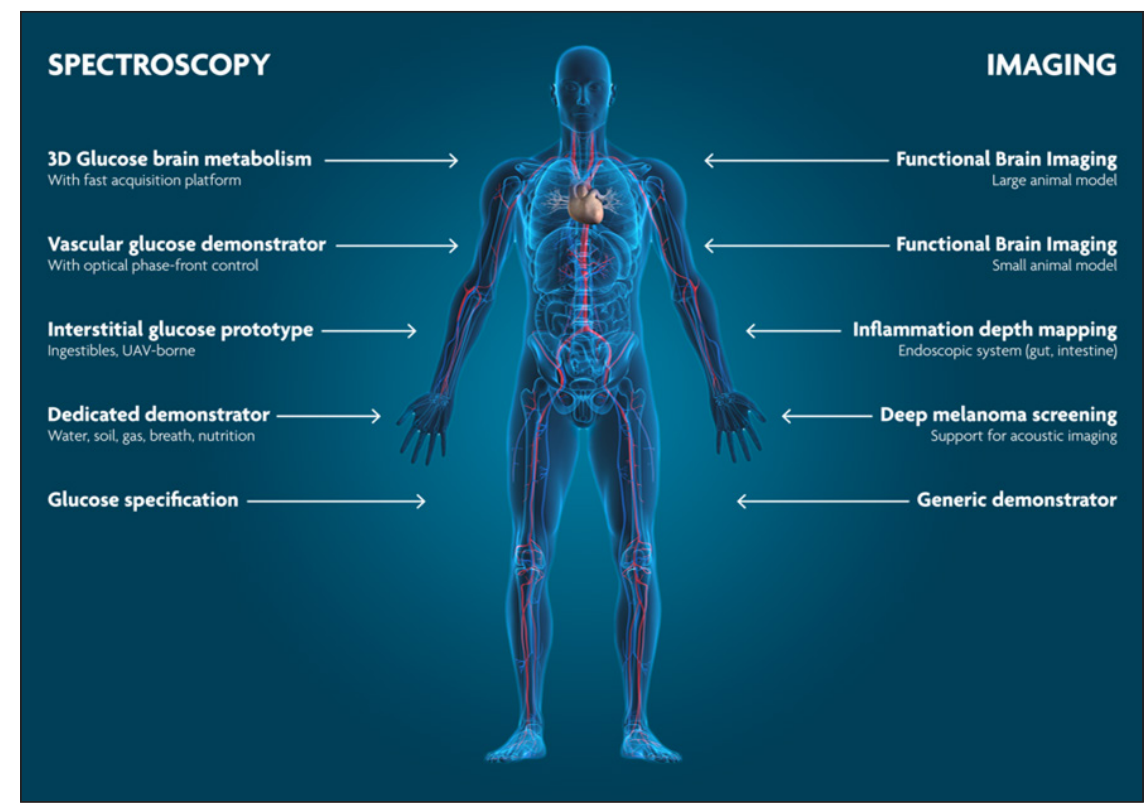

Figure 6. Photoacoustics enables non-invasive, high-resolution sensing for biomedical applications. 
VOL. 34 NO. 1 (2022)

2. A. Hermans, K. Van Gasse, J.Ø. Kjellman, C. Caër, T. Nakamura, Y. Inada, K. Hisada, T. Hirasawa, S. Cuyvers, S. Kumari, A. Marinins, R. Jansen, G. Roelkens, P. Soussan, X. Rottenberg and B. Kuyken, "High-pulse-energy III-V-on-silicon-nitride modelocked laser", APL Photonics 6, 096102 (2021). https://doi. org/10.1063/5.0058022
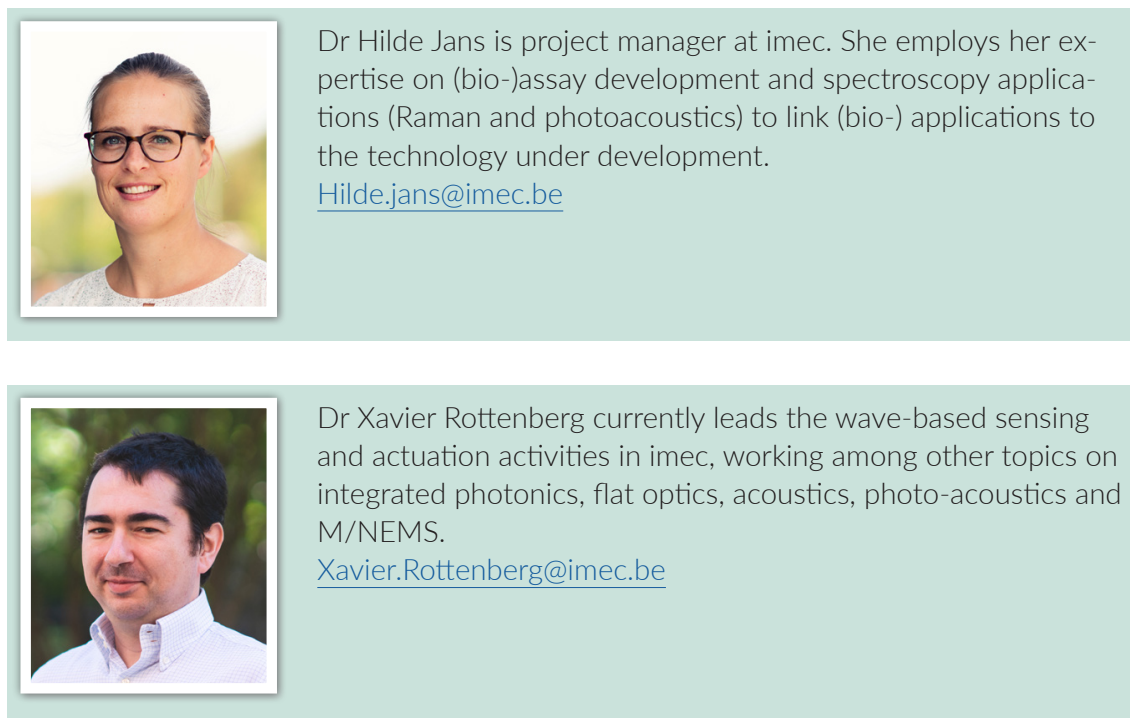